\title{
Analysis of White Matter Damage in Patients with Multiple Sclerosis via a Novel In Vivo MR Method for Measuring Myelin, Axons, and G-Ratio
}

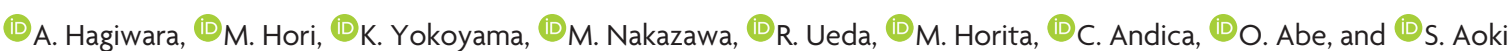

\begin{abstract}
BACKGROUND AND PURPOSE: Myelin and axon volume fractions can now be estimated via MR imaging in vivo, as can the g-ratio, which equals the ratio of the inner to the outer diameter of a nerve fiber. The purpose of this study was to evaluate WM damage in patients with MS via this novel MR imaging technique.
\end{abstract}

MATERIALS AND METHODS: Twenty patients with relapsing-remitting MS with a combined total of 149 chronic plaques were analyzed. Myelin volume fraction was calculated based on simultaneous tissue relaxometry. Intracellular and CSF compartment volume fractions were quantified via neurite orientation dispersion and density imaging. Axon volume fraction and g-ratio were calculated by combining these measurements. Myelin and axon volume fractions and g-ratio were measured in plaques, periplaque WM, and normal-appearing WM.

RESULTS: All metrics differed significantly across the 3 groups $(P<.001$, except $P=.027$ for g-ratio between periplaque WM and normal-appearing WM). Those in plaques differed most from those in normal-appearing WM. The percentage changes in plaque and periplaque WM metrics relative to normal-appearing WM were significantly larger in absolute value for myelin volume fraction than for axon volume fraction and g-ratio $(P<.001$, except $P=.033$ in periplaque WM relative to normal-appearing WM for comparison between myelin and axon volume fraction).

CONCLUSIONS: In this in vivo MR imaging study, the myelin of WM was more damaged than axons in plaques and periplaque WM of patients with MS. Myelin and axon volume fractions and g-ratio may potentially be useful for evaluating WM damage in patients with MS.

ABBREVIATIONS: AVF = axon volume fraction; EDSS = Expanded Disability Status Scale; MVF = myelin volume fraction; NAWM = normal-appearing white matter; $\mathrm{NODDI}=$ neurite orientation dispersion and density imaging; $\mathrm{PD}=$ proton density; $\mathrm{PWM}=$ periplaque white matter; Vic = volume fraction of the intracellular compartment; $\mathrm{Vec}=$ volume fraction of the extracellular compartment; Viso = volume fraction of the CSF compartment

M $\mathrm{R}$ imaging, particularly T2-weighted imaging, is used for diagnosis and follow-up in patients with MS. ${ }^{1}$ Advanced MR imaging modalities such as DTI, diffusional kurtosis imaging,

Received March 15, 2017; accepted after revision May 24.

From the Departments of Radiology (A.H., M. Hori., M.N., R.U., M. Horita, C.A., S.A.) and Neurology (K.Y.), Juntendo University School of Medicine, Tokyo, Japan; Department of Radiology (A.H., O.A.), Graduate School of Medicine, University of Tokyo, Tokyo, Japan; and Department of Radiological Sciences (R.U.), Graduate School of Human Health Sciences, Tokyo Metropolitan University, Tokyo, Japan.

This work was supported by JSPS KAKENHI grant number 16K19852; grant number JP16H06280, Grant-in-Aid for Scientific Research on Innovative Areas - Resource and technical support platforms for promoting research "Advanced Bioimaging Support"; the Japan Radiological Society and Bayer Yakuhin (KJ-08); the Impulsing Paradigm Change through disruptive Technologies (ImPACT) Program of the Council for Science, Technology and Innovation (Cabinet Office, Government of Japan); and the program for Brain Mapping by Integrated Neurotechnologies for Disease Studies (Brain/MINDS) from the Japan Agency for Medical Research and Development, AMED.

Please address correspondence to Akifumi Hagiwara, MD, Department of Radiology, Juntendo University School of Medicine, 1-2-1, Hongo, Bunkyo-ku, Tokyo, Japan, 113-8421; e-mail: a-hagiwara@juntendo.ac.jp

-- Indicates open access to non-subscribers at www.ajnr.org

http://dx.doi.org/10.3174/ajnr.A5312 $q$-space imaging, and MR spectroscopy have revealed abnormalities that were not apparent on conventional T2WI in the normalappearing white matter (NAWM $)^{2,3}$ and periplaque white matter $(\mathrm{PWM})^{4,5}$ in the brains of patients with MS. A recently developed quantification pulse sequence called QRAPMASTER (an acronym derived from "quantification of relaxation times and proton density by multiecho acquisition of a saturation-recovery by using turbo spin-echo readout") has enabled the rapid simultaneous quantification of $\mathrm{R} 1$ and $\mathrm{R} 2$ relaxation rates and proton density (PD) in approximately 5 minutes, and thus enabled the use of these measurements in clinical practice. ${ }^{6,7}$ Myelin volume fraction (MVF) can also be estimated based on R1, R2, and PD measurements. ${ }^{8}$ The model hypothesizes 4 compartments in the brain: the myelin partial volume (or MVF), the cellular partial volume, the free water partial volume, and the excess parenchymal water partial volume. It assumes that the relaxation behavior of each compartment contributes to the effective relaxation behavior of an acquisition voxel as a whole. In this model, the magnetization exchange rates between tissue compartments are 
considered to be simulated by using Bloch estimation. MVF measured by this model has been validated by myelin staining of brain sections derived from cadavers. ${ }^{9}$ We have previously reported that MVF, excess parenchymal water partial volume, R1, R2, and PD differed significantly across NAWM, PWM, and plaques. ${ }^{10}$ In plaques, these parameters differed most from those in NAWM. The percentage differences in MVF and excess parenchymal water partial volume for plaques and PWM relative to NAWM differed significantly from those of R1, R2, and PD. It was concluded that MVF and excess parenchymal water partial volume are more sensitive to the MS disease process than R1, R2, and PD.

Neurite orientation dispersion and density imaging (NODDI) is one of the recently developed advanced diffusion metrics that assumes 3 compartments: a restricted intracellular compartment (Vic), a hindered extracellular compartment (Vec), and CSF with free diffusion (Viso). ${ }^{11}$ Vic is attributed to the density of "neurites" (ie, axons and dendrites). As opposed to DTI, which is sensitive to the partial volume averaging of fiber orientations, NODDI incorporates the curving and fanning of fibers into the model and is considered to be more robust for estimating the volume of axons. ${ }^{12}$ Even though it was not explicitly designed for handling the crossing of fibers, a preliminary study has also shown the robustness of the NODDI model for crossing fibers. ${ }^{13}$

The g-ratio, which is the ratio of the inner (axon only) to the outer diameter of a myelinated axon (axon plus myelin), is associated with speed of conduction. ${ }^{14}$ Larger axons and thicker myelin sheaths give rise to faster conduction of electrochemical information, but there is a trade-off between these because of the limited space in the brain. G-ratio has a limited dynamic range in healthy $\mathrm{WM}^{13}$ and depends on age ${ }^{15-17}$ and region. ${ }^{15,18}$ Stikov et $\mathrm{al}^{12}$ developed a model that estimates "aggregate" g-ratio in a voxel via MR imaging. This model assumes that the g-ratios of all axons in a voxel are the same. Otherwise, the measured g-ratio would be the value if all of the myelin and axons were redistributed in a voxel such that all axons had the same g-ratio. Combining MVF, Viso, and Vic enables the calculation of axon volume fraction (AVF) and g-ratio. Notably, these measurements are specific to WM and not defined in GM. ${ }^{13}$ These novel MR imaging methods in combination may differentiate between demyelination and axonal degeneration, whereas conventional T2WI or FLAIR images cannot. Remyelinated lesions are known to have thinner myelin sheaths and higher g-ratios than normal tissue. ${ }^{19}$ G-ratio may also be useful for the evaluation of treatment effects in patients with MS.

The aim of the current study was to evaluate WM damage in patients with MS via novel MR imaging methods for quantifying MVF, AVF, and g-ratio.

\section{MATERIALS AND METHODS \\ Study Participants}

This prospective study recruited 24 patients with relapsing-remitting MS between April and July 2016. These patients were diagnosed according to standard criteria. ${ }^{1,20,21}$ Of these patients, 1 did not have any plaques on brain MR imaging, the images of 1 were degraded by motion artifacts, and 2 had extensive WM abnormalities and it was difficult to evaluate focal plaques correctly. Therefore, these 4 patients were excluded, and 20 patients ( 5 men and
15 women; mean age, 46.7 years; age range, 31-67 years) were included in the analysis. The median Expanded Disability Status Scale (EDSS) score ${ }^{22}$ at imaging was 1.3 (range, $0-7$ ), and the mean disease duration was $11.5 \pm 7.5$ years. The institutional review board of Juntendo University Hospital approved this study, and written informed consent was obtained from all participants.

\section{MR Imaging}

All MR imaging was performed on a 3T system (Discovery MR750w; GE Healthcare, Milwaukee, Wisconsin) with a 24channel head coil. All patients underwent MR relaxometry and diffusion-weighted, conventional T1-weighted, T2-weighted, and FLAIR imaging.

MR relaxometry was performed with a $2 \mathrm{D}$ axial pulse sequence. This is a multisection, multiecho, multisaturation delay saturation-recovery turbo spin-echo acquisition method in which images are collected with different combinations of TEs and saturation delay times. ${ }^{6}$ In Juntendo University Hospital, combinations of 2 TEs and 4 delay times were used to generate a matrix of 8 complex images that were then used to quantify longitudinal R1 relaxation and transverse $\mathrm{R} 2$ relaxation rates and $\mathrm{PD}$. The TEs used were 16.9 and $84.5 \mathrm{~ms}$, and the delay times were 146, 546, 1879 , and $3879 \mathrm{~ms}$. The TR was 4.0 seconds. The other parameters used for MR relaxometry were as follows: FOV, $240 \times 240 \mathrm{~mm}$; matrix, $320 \times 320$; echo-train length, 10; bandwidth, $31.25 \mathrm{kHz}$; section thickness/gap, $4.0 \mathrm{~mm} / 1.0 \mathrm{~mm}$; sections, 30; and acquisition time, 7 minutes 12 seconds.

With the assumption that all the R1, R2, and PD values of myelin partial volume (or MVF), excess parenchymal water partial volume, cellular partial volume, and free water partial volume contribute to the effective R1, R2, and PD in each acquisition voxel, a model was produced to estimate partial volumes of these 4 compartments as described by Warntjes et al. ${ }^{8}$ This was done by running Bloch equations and optimizing model parameters in a spatially normalized and averaged brain from a group of healthy controls. Using this model, MVF maps were created from R1, R2, and PD maps via SyMRI software (version 8.0; SyntheticMR, Linköping, Sweden). Although the more commonly used method (ie, quantitative magnetization transfer imaging) for estimating MVF to be used for calculating g-ratio requires a scaling factor to estimate MVF from measured macromolecular pool size based on proportionality, ${ }^{12}$ we omitted this procedure because the method we used directly estimates the volume fraction of myelin in a voxel. ${ }^{8}$ The R1, R2, and PD maps were also used to create synthetic T2WI with the following parameters: TR, 4500 $\mathrm{ms}$; TE, $100 \mathrm{~ms}$.

Conventional T1WI was obtained by using the following parameters: TR, $3294 \mathrm{~ms}$; TE, $18 \mathrm{~ms}$; TI, $908 \mathrm{~ms}$; FOV, $240 \times 216$ mm; matrix, $352 \times 256$; echo-train length, 8 ; section thickness/ gap, 4.0/1.0 mm; number of sections, 30. T2WI was obtained by using TR, $4500 \mathrm{~ms}$; TE, $111 \mathrm{~ms}$; FOV, $240 \times 240 \mathrm{~mm}$; matrix, $512 \times 512$; echo-train length, 24 ; bandwidth, $31.25 \mathrm{kHz}$; section thickness/gap, $4.0 \mathrm{~mm} / 1.0 \mathrm{~mm}$; and number of sections, 30 . FLAIR images were obtained by using TR, $9000 \mathrm{~ms}$; TE, $124 \mathrm{~ms}$; TI, $2472 \mathrm{~ms}$; FOV, $240 \times 240 \mathrm{~mm}$; matrix, $320 \times 224$; echo-train 


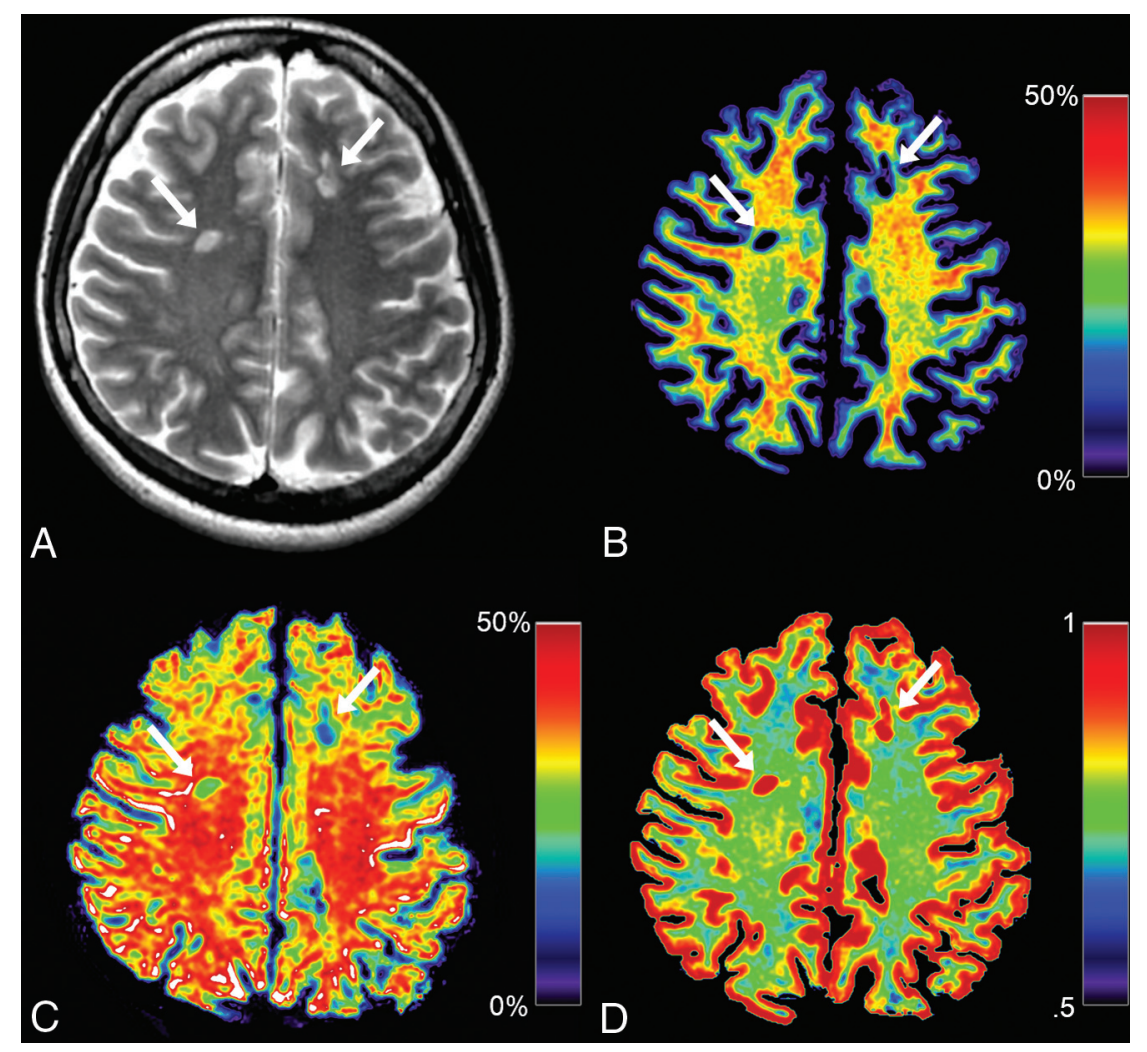

FIG 1. Representative images from a patient with MS. Synthetic T2WI $(A)$ and maps of myelin volume fraction $(B)$, axon volume fraction $(C)$, and g-ratio $(D)$ are shown. Two plaques are designated by arrows in these images. Even though myelin is severely damaged in these plaques $(B$, $5.53 \%$ and $7.23 \%$ ), the degrees of axon damage are milder (C, 31.30\% and $22.95 \%$ ). Because myelin is severely damaged in these plaques, corresponding g-ratios are close to 1.00 ( $D, 0.94$ and 0.91$)$. tropic volume fraction (Viso). Extracting free diffusion compartment Viso, remaining Vic plus extracellular volume fraction Vec equals 1. The computational procedure for NODDI was accelerated by a convex optimization procedure that converts the nonlinear fitting into a linear one. ${ }^{26}$

Maps of AVF and g-ratio were obtained as described by Stikov et al. ${ }^{12} \mathrm{Be}$ cause transverse relaxation time $\mathrm{T} 2$ of myelin water is approximately $10 \mathrm{~ms}^{27}$ and much shorter than the TE $(88.2 \mathrm{~ms}$ in this study) for acquiring sufficient diffusion sensitization, the signal of myelin is negligible in the NODDI model, but the volume of myelin is not. In other words, the volume fractions estimated by NODDI correspond to nonmyelinated tissue. Therefore, AVF is given by the following equation:

$$
A V F=(1-M V F)(1-V i s o) V i c .
$$

G-ratio is calculated by using fiber volume fraction (FVF) and AVF:

$$
A V F / F V F=g^{2} .
$$

MVF is:

$$
M V F=F V F-A V F .
$$

length, 16; section thickness/gap, 4.0/1.0 mm; and number of sections, 30 .

Single-shot echo-planar imaging was performed for DWI by using 2 b-values (30 directions with $b=1000$ seconds $/ \mathrm{mm}^{2}$ and 60 directions with $b=2000$ seconds $\left./ \mathrm{mm}^{2}\right)$. Non-DWI $(b=0$ seconds $/ \mathrm{mm}^{2}$ ) was also acquired. An array spatial sensitivity encoding technique (ASSET) with an acceleration factor of 2 was used. Other parameters used for diffusion MR imaging were as follows: TR, $5000 \mathrm{~ms}$; TE, $88.2 \mathrm{~ms}$; diffusion gradient pulse duration, $41.184 \mathrm{~ms}$; diffusion gradient separation, $29.596 \mathrm{~ms}$; FOV, $256 \times 256 \mathrm{~mm}$; matrix, $256 \times 256$; echo-train length, 128 ; bandwidth, $1953.12 \mathrm{kHz}$; section thickness/gap, $4.0 \mathrm{~mm} / 1.0 \mathrm{~mm}$; sections, 30; and acquisition time, 7 minutes 40 seconds.

For DWI, distortions caused by eddy currents and motion effects were corrected by using affine whole-brain registration to $b=0$ images with consideration of through-plane distortion in addition to in-plane distortion. ${ }^{23}$ Images were then denoised by using multishell position-orientation adaptive smoothing based on the propagation-separation approach. ${ }^{24,25}$ This algorithm does not assume a specific model and can be used for any diffusion model including NODDI. Instead of treating data from each shell separately, we treated the 2-shell diffusion data simultaneously for denoising to improve stability. ${ }^{25}$

We used the NODDI Matlab Toolbox5 (http://www.nitrc.org/ projects/noddi_toolbox) to fit the NODDI model to the acquired and processed diffusion-weighted data to create maps of intracellular volume fraction (Vic), orientation dispersion index, and iso-
Thus, g-ratio is calculated as follows:

$$
g=\sqrt{1 /[1+(M V F / A V F)]} .
$$

These calculations were performed by using an in-house program developed with Matlab (MathWorks, Natick, Massachusetts). Linear transformation was performed to register the acquired images.

\section{Image Analysis}

Synthetic T2WI and maps of MVF, AVF, and g-ratio (Fig 1) were converted to DICOM files and analyzed by using OsiriX Imaging Software, Version 7.5 (http://www.osirix-viewer.com). An experienced neuroradiologist (A.H.) used conventional and synthetic MR images to confirm 150 plaques (defined as a WM area of more than $5 \mathrm{~mm}$ in diameter in the supratentorial area with abnormally high intensity on a T2WI). Of these, 1 plaque was new compared with the MR imaging performed 2 months earlier. None of the other lesions showed remarkable change compared with the MR imaging performed at least 1 year earlier. These 149 plaques were considered chronic and were used for ROI analysis. The mean number of plaques in the 20 patients was $7.45 \pm 4.52$. A.H. manually drew freehand ROIs on plaques, PWM, and NAWM on synthetic T2WI. An ROI was placed to encircle a plaque, and up to 4 ROIs, approximately half the size of the ROI of that plaque, were also placed on the PWM (defined as a normal-intensity WM area closest to and surrounding a plaque) (Fig 2). ${ }^{4,5,10}$ The PWM ROIs were placed approximately $90^{\circ}$ from the adjacent ROI to form a 


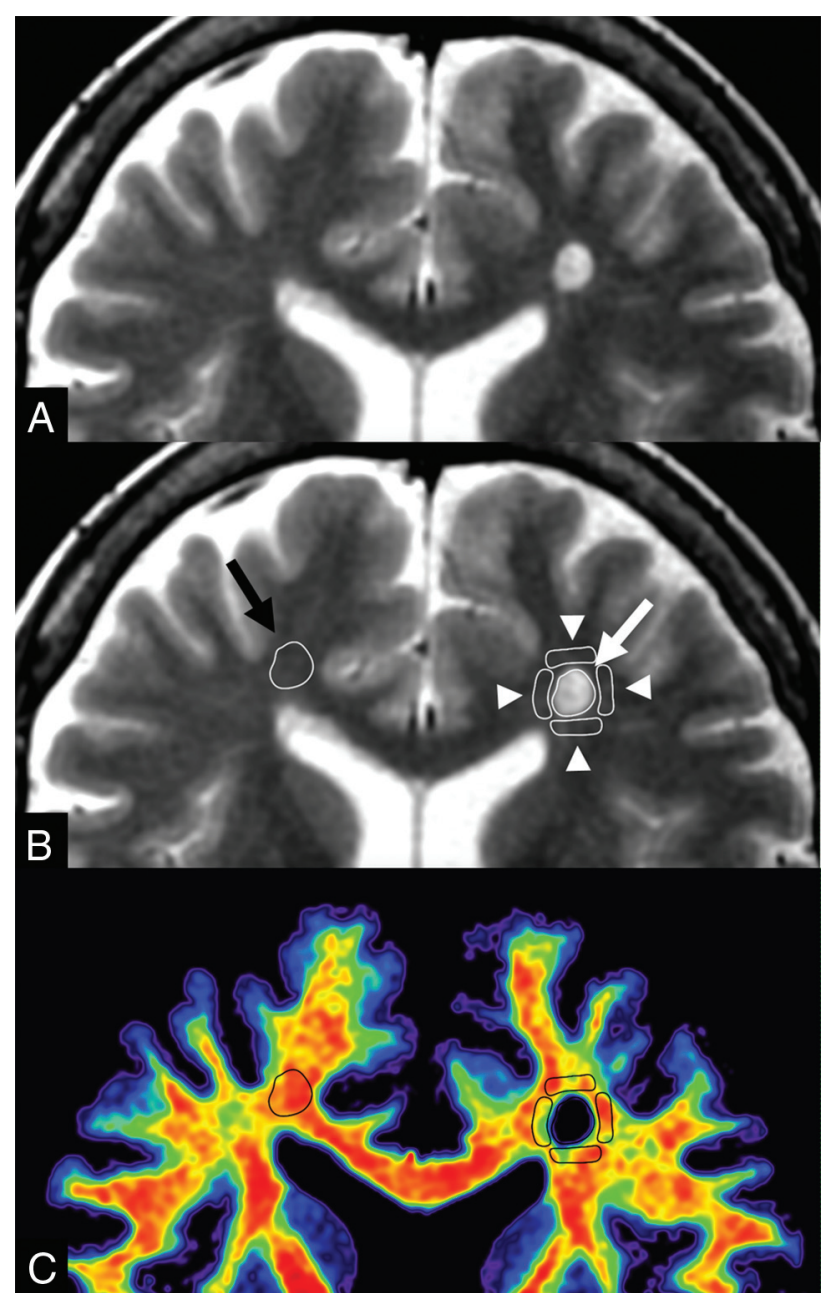

FIG 2. An example of ROI analysis in a patient with MS. The upper 2 images show the same synthetic T2WI without $(A)$ and with $(B)$ the placement of ROIs. A ROI (white arrow) was drawn on a plaque, and 4 ROIs (arrowheads) were placed on periplaque white matter to encircle the plaque. The ROI of the plaque was copied and pasted onto the contralateral normal-appearing white matter (black arrow). These ROls were then copied and pasted onto maps of myelin volume fraction, axon volume fraction, and g-ratio. A map of the corresponding myelin volume fraction $(C)$ is shown as an example.

box that surrounded the plaque. If a PWM ROI overlapped CSF, GM, or other plaques, it was discarded; 206 PWM ROIs were removed on this basis (ie, 390 PWM ROIs were used for analysis). The ROI of the plaque was copied and pasted on the contralateral NAWM. The mean ROI sizes were $45.17 \pm 30.19 \mathrm{~mm}^{2}$ for plaques and $22.72 \pm 15.16 \mathrm{~mm}^{2}$ for PWM. All the ROIs made on synthetic T2WI were copied and pasted onto the MVF, AVF, and g-ratio maps for the same patient, and the mean value of each ROI was recorded. To confirm the accuracy of ROI analyses, a second investigator (M. Horita) blinded to the patient information also independently conducted the same measurements.

\section{Statistical Analysis}

Because not all data were normally distributed, we used the SteelDwass test for multiple comparisons to compare MVF, AVF, and g-ratio values among plaques, PWM, and NAWM. The percentage changes of plaques or PWM relative to NAWM were also calculated and compared among different metrics (ie, MVF, AVF,
Table 1: Descriptive values of plaques, PWM, and NAWM

\begin{tabular}{lccc}
\hline & MVF (\%) & AVF (\%) & G-Ratio (\%) \\
\hline Plaques & $5.97 \pm 4.86$ & $31.49 \pm 10.39$ & $0.93 \pm 0.058$ \\
PWM & $25.74 \pm 4.42$ & $39.08 \pm 7.09$ & $0.77 \pm 0.049$ \\
NAWM & $30.96 \pm 3.74$ & $44.10 \pm 7.56$ & $0.76 \pm 0.051$ \\
\hline
\end{tabular}

${ }^{a}$ Values are mean \pm SD. $P<.001$ for all metrics among each tissue type, except $P=$ .027 for g-ratio between PWM and NAWM.

Table 2: Percentage changes of MVF, AVF, and g-ratio in plaques and PWM relative to NAWM

\begin{tabular}{lrrr}
\hline & \multicolumn{1}{c}{ MVF (\%) } & \multicolumn{1}{c}{ AVF (\%) } & \multicolumn{1}{c}{ G-Ratio (\%) $^{\mathbf{a}}$} \\
\hline Plaques & $80.78 \pm 15.27$ & $27.18 \pm 25.27$ & $23.81 \pm 11.52$ \\
PWM & $16.19 \pm 14.82$ & $9.31 \pm 22.05$ & $2.17 \pm 8.24$
\end{tabular}

${ }^{a}$ Values are mean \pm SD. $P<.001$ for percentage changes of plaques and PWM relative to NAWM for comparisons among all metrics, except $P=.016$ for percentage change of plaques relative to NAWM when comparing between AVF and g-ratio, and $P=.033$ for percentage change of PWM relative to NAWM when comparing between MVF and AVF.

and g-ratio). The signs of the percentage changes for MVF and AVF were inverted for statistical analysis because these values were higher overall in NAWM than in plaques and PWM. EDSS and disease duration were correlated with MVF, AVF, and g-ratio of NAWM averaged in each patient via the Spearman rank order correlation coefficient. A 2 -sided $P$ value $<.05$ was considered significant. All statistical analyses were performed with the software package R, version 3.2.1 (http://www.r-project.org/).

\section{RESULTS}

The results of ROI analysis and comparisons among plaques, PWM, and NAWM are shown in Table 1. MVF, AVF, and g-ratio all differed significantly among plaques, PWM, and NAWM. MVF and AVF were lower in plaques and PWM than in NAWM, with plaques showing the lowest value $(P<.001)$, and g-ratio was higher in plaques and PWM than in NAWM, with plaques showing the highest value $(P<.001$, except $P=.027$ for g-ratio between PWM and NAWM).

The percentage changes of MVF, AVF, and g-ratio in plaques and PWM relative to NAWM are shown in Table 2. Those of MVF and AVF in plaques relative to NAWM differed significantly more from zero than that of g-ratio, with that of MVF differing the most from zero $(P<.001$, except $P=.016$ for comparison between those of AVF and g-ratio). Those of MVF and AVF in PWM relative to NAWM differed significantly more from zero than that of g-ratio, with that of MVF differing the most from zero $(P<.001$, except $P=.033$ for comparison between those of MVF and AVF).

The interobserver reproducibility between the 2 observers (A.H. and M. Horita) for the mean MVF values was measured. The interclass correlation coefficient for plaques was 0.84 (95\% CI, 0.780.88 ), that for PWM was 0.94 (95\% CI, 0.92-0.96), and that for NAWM was 0.89 (95\% CI, 0.85-0.92).

Significant correlations with EDSS and disease duration were not found for MVF, AVF, and g-ratio of NAWM averaged in each patient (EDSS versus MVF, $P=.25$; EDSS versus AVF, $P=.87$; EDSS versus g-ratio, $P=.80$; disease duration versus MVF, $P=$ .25 ; disease duration versus AVF, $P=.26$; disease duration versus g-ratio, $P=.22$ ).

AJNR Am J Neuroradiol 38:1934-40 Oct 2017 www.ajnr.org 


\section{DISCUSSION}

The observation of lower MVF in plaques and PWM than in NAWM, with plaques showing the lowest value, is consistent with the results of our previous report. ${ }^{10}$ In this study, AVF of PWM was also in between that of NAWM and plaques, with plaques showing the lowest value. However, the percentage changes of MVF for plaques and PWM relative to NAWM were higher than those of AVF. This implies that myelin was more damaged than axons in plaques and PWM. This corresponds to the results of previous histologic studies showing that demyelination is the primary event in patients with MS, and axons are relatively preserved in chronic plaques. ${ }^{28}$ Even though Wallerian degeneration is known to cause axonal loss and contribute to secondary myelin degradation in PWM, ${ }^{29,30}$ myelin loss may be the primary event in PWM as well as in plaques. ${ }^{28}$ However, there is growing evidence that axonal damage is also a feature of MS that is associated with long-term disability and functional deficits. ${ }^{31}$ For example, the average percentage change of AVF in plaques relative to NAWM in our study was $27.18 \%$, lower than the value $(68 \%)$ reported by Bjartmar et $\mathrm{al}^{32}$ for histopathologic average axonal loss in MS lesions. However, their study involved the spinal cords of 5 paralyzed patients with MS with EDSS scores of $\geq 7.5$, whereas the median EDSS score of the patients in our study was much lower (1.3), and the degrees of axonal damage in the patients in the 2 studies were presumably different. A greater number of patients would be required to stratify them according to EDSS to investigate patterns of myelin and axonal damage according to disease progression. In the current study, neither EDSS nor disease duration were significantly correlated with MVF, AVF, or g-ratio in NAWM. Correlations between clinical indicators and MVF, AVF, and g-ratio should also be re-evaluated in a larger study.

Our study is the first to report the g-ratio in the brains of a number of patients with MS. The average g-ratio of 0.76 in NAWM of patients with MS in this study is almost equal to the value of 0.75 in a patient with MS measured via MR imaging reported by Stikov et al. ${ }^{12}$ In the current study, the average g-ratio of MS plaques was higher than that of NAWM ( 0.93 versus 0.76 ). This is consistent with a histologic study that showed myelin is thinner in plaques even after partial remyelination. ${ }^{17}$ Although our result for the relationship of g-ratios between MS plaques and NAWM corresponds to the aforementioned report by Stikov et $\mathrm{al},{ }^{12}$ the reported average g-ratio for plaques was 0.80 in that study, which is lower than that of the current study (0.93). This difference in g-ratios may be related to variations in the disease duration and differences in the methods used to measure g-ratio. In the current study, g-ratios in various regions were averaged, and the dependence of g-ratio on specific regions was not explored. Larger axons (eg, those in corticospinal tracts) are known to have greater g-ratios than smaller axons. ${ }^{18,33}$

There are various approaches for estimating MVF in the brain. Magnetization transfer imaging is one of these, and quantitative magnetization transfer imaging has shown a strong correlation with quantitative histology. ${ }^{34} \mathrm{MR}$ studies evaluating g-ratio have mostly been based on magnetization transfer imaging. ${ }^{12,15,18}$ However, quantitative magnetization transfer imaging requires approximately 30 minutes for acquisition, and it is still unrealistic for routine use in a clinical setting. Quantitative magnetization transfer imaging first measures macromolecular pool size, and a coefficient for regressing it to MVF should be determined in each protocol. ${ }^{12}$ The model based on tissue relaxometry used in the current study $^{8}$ directly estimates MVF in a voxel and can be used without determining a coefficient as is done by quantitative magnetization transfer imaging. Our report is the first to show g-ratios calculated with MVF measured via simultaneous tissue relaxometry. Although MVF calculated based on this tissue relaxometry has been validated in cadavers with no known brain diseases, ${ }^{9}$ the methodology has not yet been histologically validated in brains with MS. In plaques, expected histology is heterogeneous, including myelin debris, poor remyelination, and remyelination with higher quality. ${ }^{35}$ The extent to which MVF is affected by myelin debris may depend on the MR imaging technique used for estimation. $^{13}$

The myelin model used in our study considers magnetization exchange rates between tissue compartments. ${ }^{8}$ However, it does not explicitly incorporate a partial volume pool to account for magnetization transfer effects, which were shown to create an offset bias for steady-state approaches. ${ }^{36}$ Magnetization transfer effects may result in small changes of our observed Carr-Purcell Meiboom-Gill multiecho signal amplitudes, which will propagate to the R2 estimations. In the model used in our study, however, myelin is estimated from combinations of the measured R1, R2, and PD values, and the effect of a potential offset in R2 is expected to be small. The inclusion of magnetization transfer effects and correction of a potential bias on our myelin water fraction measurements may be improvements in future works. Mossahebi et $\mathrm{al}^{37}$ recently suggested a method to reduce the partial volume effect by CSF on the estimation of magnetization transfer. CSF partial volume is expected at the brain tissue interface with the surrounding CSF and is accounted for in the model used in our study by the incorporation of the free water partial volume compartment.

In addition to calculating MVF, measures of R1, R2, and PD acquired via simultaneous tissue relaxometry can also be used for synthesizing various contrast-weighted images, including T1weighted, T2-weighted, FLAIR, double inversion recovery, or phase-sensitive inversion recovery images. ${ }^{7,38-40}$ Thus, the need to obtain these images separately can be eliminated. The clinical usefulness of this specific tissue relaxometry with synthetic MR imaging has been shown in studies of patients with MS. ${ }^{41,42}$

The current study had some limitations. First, the sample size was small. Second, it did not include healthy controls. Third, only chronic plaques were investigated, and acute plaques may yield different results. Fourth, because the resolution of 2 techniques used for simultaneous tissue relaxometry and NODDI were not matched, there was a possibility of misregistration and partial volume effects.

\section{CONCLUSIONS}

This in vivo MR imaging study showed myelin to be more damaged than axons in plaques and PWM of patients with MS. The average g-ratio of NAWM was similar to a previously reported value $^{12}$ based on a single patient with MS, but that of plaques was higher than the value reported in that prior study, which was 
measured by methods other than those used in the current study. MVF, AVF, and g-ratio have the potential to be useful for evaluating WM damage in patients with MS.

\section{ACKNOWLEDGMENTS}

We thank Araya Brain Imaging for technical support with data analysis. We are grateful to Dr. Marcel Warntjes and Dr. Nikola Stikov for providing us with the idea support and thoughtful comments on our manuscript.

Disclosures: Akifumi Hagiwara—RELATED: Grants/Grants Pending: JSPS KAKENHI, Advanced Bioimaging Support, Japan Radiological Society and Bayer Yakuhin, ImPACT Program, AMED, Comments: this work was supported by JSPS KAKENHI Grant Number 16K19852; grant number JP16H06280, Grant-in-Aid for Scientific Research on Innovative Areas - Resource and technical support platforms for promoting research "Advanced Bioimaging Support"; the Japan Radiological Society and Bayer Yakuhin (KJ-08); the Impulsing Paradigm Change through disruptive Technologies (ImPACT) Program of the Council for Science, Technology and Innovation (Cabinet Office, Government of Japan); and the program for Brain Mapping by Integrated Neurotechnologies for Disease Studies (Brain/MINDS) from the Japan Agency for Medical Research and development, AMED. Kazumasa Yokoyama—UNRELATED: Other: belonging to the department funded by Mitsubishi Tanabe Pharma, Hydrogen Health Medical Labo, ABIST, Melodian, Kenkoukazoku, Yamato, Biogen Idec Japan, Bayer, Nihon Pharmaceutical, Asahi Kasei Medical, Japan Blood Products Organization, MiZ, Ono Pharmaceutical.* Shigeki Aoki-RELATED: Grant: Brain/MINDS, ABiS*; UNRELATED: Board Membership: Bayer; Consulting Fee or Honorarium: SyMRI meeting chair; Grants/Grants Pending: Bayer, Daiichi-Sankyo, Eisai, Fuji-yakuhin, Fuji Film RI, Mediphysics, Toshiba, Comments: grant for diagnostic radiology*; Payment for Lectures (including service on Speakers Bureaus): GE, Toshiba, Hitachi, Siemens, Bayer, Daiichisankyo, Eisai, Fuji-yakuhin, Fuji Film RI, Mediphysics, Novartis, Takeda, Biojen, Comments: honorarium for lectures/chair. *Money paid to the institution.

\section{REFERENCES}

1. Polman CH, Reingold SC, Banwell B, et al. Diagnostic criteria for multiple sclerosis: 2010 revisions to the McDonald criteria. Ann Neurol 2011;69:292-302 CrossRef Medline

2. Filippi M, Rocca MA, De Stefano N, et al. Magnetic resonance techniques in multiple sclerosis: the present and the future. Arch Neurol 2011;68:1514-20 CrossRef Medline

3. Yoshida M, Hori M, Yokoyama K, et al. Diffusional kurtosis imaging of normal-appearing white matter in multiple sclerosis: preliminary clinical experience. Jpn J Radiol 2013;31:50-55 CrossRef Medline

4. Guo AC, MacFall JR, Provenzale JM. Multiple sclerosis: diffusion tensor MR imaging for evaluation of normal-appearing white matter. Radiology 2002;222:729-36 CrossRef Medline

5. Hori M, Yoshida M, Yokoyama K, et al. Multiple sclerosis: benefits of $q$-space imaging in evaluation of normal-appearing and periplaque white matter. Magn Reson Imaging 2014;32:625-29 CrossRef Medline

6. Warntjes JB, Leinhard OD, West J, et al. Rapid magnetic resonance quantification on the brain: optimization for clinical usage. Magn Reson Med 2008;60:320-29 CrossRef Medline

7. Hagiwara A, Warntjes M, Hori M, et al. SyMRI of the brain: rapid quantification of relaxation rates and proton density, with synthetic MRI, automatic brain segmentation, and myelin measurement. Invest Radiol 2017 Mar 3. [Epub ahead of print] CrossRef Medline

8. Warntjes M, Engström M, Tisell A, et al. Modeling the presence of myelin and edema in the brain based on multi-parametric quantitative MRI. Front Neurol 2016;7:16 CrossRef Medline

9. Warntjes JBM, Persson A, Berge J, et al. Myelin detection using rapid quantitative MR imaging correlated to macroscopically registered luxol fast blue-stained brain specimens. AJNR Am J Neuroradiol 2017;38:1096-1102 CrossRef Medline

10. Hagiwara A, Hori M, Yokoyama K, et al. Utility of a multiparametric quantitative MRI model that assesses myelin and edema for evaluating plaques, periplaque white matter, and normal-appearing white matter in patients with multiple sclerosis: a feasibility study. AJNR Am J Neuroradiol 2017;38:237-42 CrossRef Medline

11. Zhang H, Schneider T, Wheeler-Kingshott CA, et al. NODDI: practical in vivo neurite orientation dispersion and density imaging of the human brain. Neuroimage 2012;61:1000-16 CrossRef Medline

12. Stikov N, Campbell JS, Stroh T, et al. In vivo histology of the myelin g-ratio with magnetic resonance imaging. Neuroimage 2015;118: 397-405 CrossRef Medline

13. Campbell JS, Leppert IR, Narayanan S, et al. Promise and pitfalls of g-ratio estimation with MRI. arXiv:1701.02760v1 [physics.med-ph]. January 10, 2017. https://arxiv.org/abs/1701.02760v1. Accessed July 2, 2017

14. Rushton WA. A theory of the effects of fibre size in medullated nerve. J Physiol 1951;115:101-22 CrossRef Medline

15. Cercignani M, Giulietti G, Dowell NG, et al. Characterizing axonal myelination within the healthy population: a tract-by-tract mapping of effects of age and gender on the fiber g-ratio. Neurobiol Aging 2017;49:109-18 CrossRef Medline

16. Dean DC 3rd, O'Muircheartaigh J, Dirks H, et al. Mapping an index of the myelin g-ratio in infants using magnetic resonance imaging. Neuroimage 2016;132:225-37 CrossRef Medline

17. Melbourne A, Eaton-Rosen Z, Orasanu E, et al. Longitudinal development in the preterm thalamus and posterior white matter: MRI correlations between diffusion weighted imaging and $\mathrm{T} 2$ relaxometry. Hum Brain Mapp 2016;37:2479-92 CrossRef Medline

18. Mohammadi S, Carey D, Dick F, et al. Whole-brain in-vivo measurements of the axonal g-ratio in a group of 37 healthy volunteers. Front Neurosci 2015;9:441 Medline

19. Albert M, Antel J, Brück W, et al. Extensive cortical remyelination in patients with chronic multiple sclerosis. Brain Pathol 2007;17: 129-38 CrossRef Medline

20. McDonald WI, Compston A, Edan G, et al. Recommended diagnostic criteria for multiple sclerosis: guidelines from the International Panel on the diagnosis of multiple sclerosis. Ann Neurol 2001;50: 121-27 CrossRef Medline

21. Polman CH, Reingold SC, Edan G, et al. Diagnostic criteria for multiple sclerosis: 2005 revisions to the "McDonald Criteria." Ann Neurol 2005;58:840-46 CrossRef Medline

22. Kurtzke JF. A new scale for evaluating disability in multiple sclerosis. Neurology 1955;5:580-83 CrossRef Medline

23. Mohammadi S, Möller HE, Kugel H, et al. Correcting eddy current and motion effects by affine whole-brain registrations: evaluation of three-dimensional distortions and comparison with slicewise correction. Magn Reson Med 2010;64:1047-56 CrossRef Medline

24. Becker SM, Tabelow K, Voss HU, et al. Position-orientation adaptive smoothing of diffusion weighted magnetic resonance data (POAS). Med Image Anal 2012;16:1142-55 CrossRef Medline

25. Becker SM, Tabelow K, Mohammadi S, et al. Adaptive smoothing of multi-shell diffusion weighted magnetic resonance data by msPOAS. Neuroimage 2014;95:90-105 CrossRef Medline

26. Daducci A, Canales-Rodríguez EJ, Zhang H, et al. Accelerated microstructure imaging via convex optimization (AMICO) from diffusion MRI data. Neuroimage 2015;105:32-44 CrossRef Medline

27. Levesque IR, Pike GB. Characterizing healthy and diseased white matter using quantitative magnetization transfer and multicomponent $\mathrm{T}(2)$ relaxometry: a unified view via a four-pool model. Magn Reson Med 2009;62:1487-96 CrossRef Medline

28. Lucchinetti CF, Brück W, Rodriguez M, et al. Distinct patterns of multiple sclerosis pathology indicates heterogeneity on pathogenesis. Brain Pathol 1996;6:259-74 CrossRef Medline

29. Dziedzic T, Metz I, Dallenga T, et al. Wallerian degeneration: a major component of early axonal pathology in multiple sclerosis. Brain Pathol 2010;20:976-85 Medline

30. Simons M, Misgeld T, Kerschensteiner M. A unified cell biological perspective on axon-myelin injury. J Cell Biol 2014;206:335-45 CrossRef Medline

31. Haines JD, Inglese M, Casaccia P. Axonal damage in multiple sclerosis. Mt Sinai J Med 2011;78:231-43 CrossRef Medline

32. Bjartmar C, Kidd G, Mörk S, et al. Neurological disability correlates 
with spinal cord axonal loss and reduced $\mathrm{N}$-acetyl aspartate in chronic multiple sclerosis patients. Ann Neurol 2000;48:893-901 Medline

33. Graf von Keyserlingk D, Schramm U. Diameter of axons and thickness of myelin sheaths of the pyramidal tract fibres in the adult human medullary pyramid. Anat Anz 1984;157:97-111 Medline

34. West KL, Kelm ND, Carson RP, et al. Myelin volume fraction imaging with MRI. Neuroimage 2016 Dec 23. [Epub ahead of print] CrossRef Medline

35. Lampron A, Larochelle A, Laflamme N, et al. Inefficient clearance of myelin debris by microglia impairs remyelinating processes. $J$ Exp Med 2015;212:481-95 CrossRef Medline

36. Liu F, Block WF, Kijowski R, et al. Rapid multicomponent relaxometry in steady state with correction of magnetization transfer effects. Magn Reson Med 2016;75:1423-33 CrossRef Medline

37. Mossahebi P, Alexander AL, Field AS, et al. Removal of cerebrospinal fluid partial volume effects in quantitative magnetization transfer imaging using a three-pool model with nonexchanging water component. Magn Reson Med 2015;74:1317-26 CrossRef Medline
38. Hagiwara A, Nakazawa M, Andica C, et al. Dural enhancement in a patient with Sturge-Weber syndrome revealed by double inversion recovery contrast using synthetic MRI. Magn Reson Med Sci 2016; 15:151-52 CrossRef Medline

39. Hagiwara A, Hori M, Suzuki M, et al. Contrast-enhanced synthetic MRI for the detection of brain metastases. Acta Radiol Open 2016;5: 2058460115626757 CrossRef Medline

40. Andica C, Hagiwara A, Nakazawa M, et al. The advantage of synthetic MRI for the visualization of early white matter change in an infant with Sturge-Weber syndrome. Magn Reson Med Sci 2016;15: 347-48 CrossRef Medline

41. Granberg T, Uppman M, Hashim F, et al. Clinical feasibility of synthetic MRI in multiple sclerosis: a diagnostic and volumetric validation study. AJNR Am J Neuroradiol 2016;37:1023-29 CrossRef Medline

42. Hagiwara A, Hori M, Yokoyama K, et al. Synthetic MRI in the detection of multiple sclerosis plaques. AJNR Am J Neuroradiol 2017;38: 257-63 CrossRef Medline 